\title{
Photon and its hadronic interaction
}

\author{
Maria Krawczyk ${ }^{\mathrm{a}}$ \\ ${ }^{a}$ Institute of Theoretical Physics, Warsaw University, \\ ul. Hoża 69, 00-681 Warsaw, Poland
}

A short overview of basics aspects of hadronic interaction of the photon is presented.

\section{Introduction}

Photon is one of the oldest elementary particle with very well known (QED) properties, and as such it is considered to be a tool ("ideal probe") to test the structure of more complicated objects like a proton. For example, in the DIS sidered as the main source of information on the inner structure of the proton, the structure functions $F_{1,2, L}^{\text {proton }}$ for the proton can be measured. The probe - in a wide range of $Q^{2}$ - is provided by flux of virtual photons, emitted by primary electrons (positrons). So, besides proton also electron

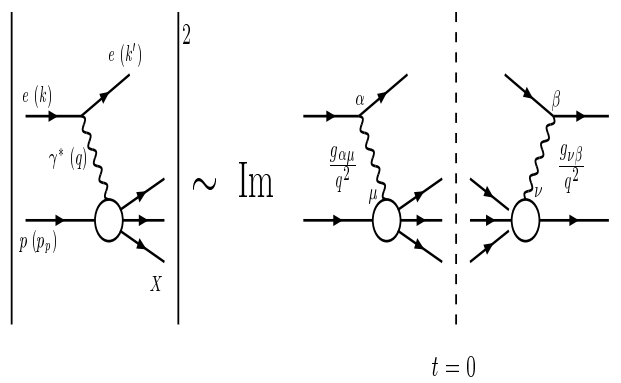

and photon are involved in the measurement of the structure functions and the parton densities of the proton. A factorization allows to treat independently the electron and proton part (figure above). The contributions of the longitudinal (L) and transverse $(\mathrm{T})$ virtual photons can also be separated. Therefore, we can write

$$
d \sigma^{e p \rightarrow e X}=\Gamma_{T} d \sigma^{\gamma_{T}^{*} p \rightarrow X}+\Gamma_{L} d \sigma^{\gamma_{L}^{*} p \rightarrow X} .
$$

Note, that the structure functions $F_{1,2, L}^{\text {proton }}$ are proportional to the combinations of the cross sections for processes $\gamma_{T, L}^{*} p \rightarrow X$. One can ask if the photon is only a neutral probe in such measurements and to what extend its apparent "struc- ture" influences the corresponding structure functions of the proton?

Effects of the "structure" of the photon may influence our knowledge of the partonic content of the proton also in the large- $p_{T}$ photoproduction at HERA. However here both the initial (almost real) photon and the initial proton are on equal footing and it is natural nowdays to take into account partonic content of both of them.

Similarly, the DIS events at HERA, if correspond to the large- $p_{T}$ single particle or jet production for $p_{T}^{2} \gg Q^{2}$, are sensitive to the "structure" of the virtual photon (with virtuality $Q^{2}$ ).

It is clear, that if we want to understand a proton, we should understand a photon itself. It is interesting to realized that approximately at the same time (1922-3) we have learned that the proton is not a fundamental particle, while the photon is such a particle (Compton effect). Since early sixties XX we realized that the photon in fact belongs to a "hadron family" with the closest relatives (according to the VMD idea)- the vector mesons: $\rho, \omega$ and $\phi$. Next, the notion of a partonic content of $\gamma$ have been introduced to describe in an effective way its hadronic interaction.

\section{Photon}

At present colliders testing the partonic content of the photon is a difficult task, as at both LEP and HERA the photon is not a primary particle. The question if it is simpler to deal with a partonic content of the electron was addressed during this workshop.

In the inclusive process $e^{+} e^{-} \rightarrow e^{+} e^{-}$hadrons one can probe the "structure" of photon in various ways depending on the virtualities of emitted 
photons, $\left|q_{1}^{2}\right|=Q^{2}$ and $\left|q_{2}^{2}\right|=P^{2}$ (figure below): - The region $Q^{2} \gg P^{2} \gg \Lambda_{Q C D}^{2}$ corresponds to $\mathrm{DIS}_{e \gamma^{*}}$, where one can measure the structure functions of the virtual photon.

- For $Q^{2} \gg \Lambda_{Q C D}^{2}, P^{2} \sim 0$ the corresponding process is $\mathrm{DIS}_{e \gamma}$, where one can measure the structure functions of the real photon.

- For $Q^{2} \sim P^{2} \sim 0$ the total cross section $\sigma_{\gamma \gamma}^{t o t}$ can be studied.

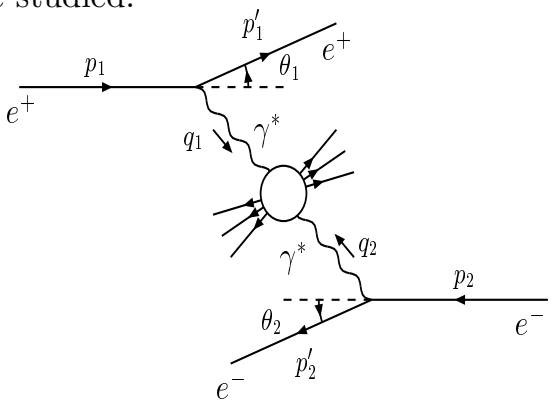

In the DIS events $e \gamma \rightarrow e X$ various structure functions for the real photons can be measured in principle, however data are mainly for $F_{2}^{\gamma}$.

The $F_{2}^{\gamma}$ structure function can be related to the partonic densities of the real photon. In the Parton Model (PM) one obtains a prediction from a pure QED that the quark densities $q^{\gamma} \sim \ln Q^{2}$, and that $q^{\gamma}$ are large at the large value of $x$. In QCD there are leading $\ln Q^{2}$ corrections which can be sum up to all orders using an inhomogeneous DGLAP evolution equations. This form of equation allows to obtain an asymptotic solutions for quark densities in the photon without any input. In practice, one use an input usually based on VMD (sometimes modelled by $\pi$ (sic!)).

In many analyses the quark densities in $\gamma$ are treated as being proportional to $\alpha / \alpha_{s}$, while in others to $\alpha$, since the main $\ln Q^{2}$-dependence mentioned above has nothing to do with QCD and $\alpha_{s}$. The way of counting has practical consequences as it influences the choice of diagrams which define the NLO set.

The cross section for the large- $p_{T}$ hadronic processes involving photons get contributions from diagrams with the direct and the resolved photon interaction with quarks. Note, that the resolvedphoton contribution are possible for real and virtual photons, in the latter case provided that $p_{T}^{2}$ is larger that $P^{2}$ - a "virtuality" of the $\gamma^{*}$. Note that for the resolved virtual photons processes one should take into account not only cross section for a defined polarization of $\gamma^{*}(\mathrm{~L}$ or $\mathrm{T})$ but also, since $\gamma$ is not a primary particle, the corresponding interference terms: LT and T1 T2.

\section{New results for proton and photon}

There many new data for the proton from HERA and for photon from the LEP (DIS and "large- $p_{T}$ " events) and from HERA ("large- $p_{T}$ events"), as presented at this workshop.

There is an impressive theoretical progress in the construction of the parton parametrizations for the proton with a higher precision and using various evolution equations, DGLAP, BFKL and CCFM. Of a great importance are special treatments of the heavy-quark thresholds and error analyses of parton densities preformed recently. This stimulates the corresponding progress for a photon, reported during workshop.

\section{Old and new ideas}

- In 1934 L. de Broglie considered "The Neutrino Theory of Light", with the light quanta composed of $\nu \bar{\nu}$ pairs (also Pauli, Heisenberg, Jordan, Kronig, Born). No satisfactory derivation of Bose statistics of light quanta from Fermi statistics for $\nu$ was found and this idea was abandoned (almost).

- The new idea is a Non-Commutative QED with a selfinteraction of photons.

\section{Instead of a summary}

The study of light has resulted in achievements of insight, imagination and ingenuity unsurpassed in any field of mental activity; it illustrates, too, better than any other branch of physics, the Vicissitudes of theories (Sir J.J. Thomson, 1925).

For future study a Photon Collider with a real photon as a primary particle will be very useful.

Acknowledgment: I would like to thank the Local Organizing Committee for this excellent workshop and all forms of support during my stay in Frascati. 